\title{
Análise da Dispersão do Intervalo QT em Pacientes com Infarto Agudo do Miocárdio: Efeito da Reperfusão Coronariana
}

\author{
QT Interval Dispersion Analysis in Acute Myocardial Infarction Patients: Coronary \\ Reperfusion Effect
}

Neuza Helena Moreira Lopes, César Grupi, Cleberson H. Dina, Aécio F. T. de Gois, Ludhmila A. Hajjar, Beatriz Ayub, Carlos Eduardo Rochitte, José Antonio Franchini Ramires, Whady A. Hueb, Roberto Kalil Instituto do Coração do Hospital das Clínicas - FMUSP - São Paulo, SP

\section{Objetivo}

Estudar o efeito da reperfusão precoce da artéria relacionada ao infarto sobre a dispersão do intervalo $\mathrm{QT}(\Delta \mathrm{QT})$, e seu valor como marcador de reperfusão coronária e de arritmias ventriculares.

\section{Métodos}

Foram avaliados 106 pacientes com reperfusão (CR) e 48 pacientes sem reperfusão (SR) que receberam terapia trombolítica na fase aguda do infarto. Foram analisados os eletrocardiogramas realizados na admissão e no $4^{\circ}$ dia de evolução. A $\Delta Q T$, definido como a diferença entre o maior e o menor intervalo QT, foram medidos no ECG de 12 derivações.

\section{Resultados}

$\mathrm{Na}$ evolução do grupo com reperfusão, houve redução significativa da $\Delta Q T$ de $89,66 \pm 20,47 \mathrm{~ms}$ para $70,95 \pm 21,65 \mathrm{~ms}(\mathrm{p}<0,001)$. Por outro lado, no grupo sem reperfusão, houve aumento significativo da $\Delta Q T$ de $81,27 \pm 20,52 \mathrm{~ms}$ para $91,85 \pm 24,66 \mathrm{~ms}$ ( $p<0,001$ ). Análise de regressão logística demonstrou que a magnitude de redução entre a $\Delta Q T$ pré e pós-trombólise foi o fator independente que identificou mais efetivamente a reperfusão coronária (OR $1,045, p<0,0001$; IC $95 \%$ ). Não houve diferença significativa das medidas de dispersão quando comparados os pacientes que apresentaram arritmias ventriculares nas primeiras $48 \mathrm{~h}$ com aqueles sem arritmias.

\section{Conclusão}

Esse estudo mostra que a $\triangle Q T$ reduz significativamente em pacientes com infarto agudo do miocárdio submetidos à trombólise com sucesso, aumentando nos pacientes que evoluem com a artéria fechada. A redução $\Delta Q T$ entre a situação pré e pós-trombólise foi fator preditor de reperfusão coronária nesses pacientes, não apresentando correlação com arritmias ventriculares.

\section{Palavras-chave}

Dispersão do QT, infarto agudo do miocárdio, reperfusão coronariana.

\section{Objective}

To study the effect of early reperfusion of infarctrelated artery on $Q T(\triangle Q T)$ dispersion interval, as well as how valuable it is as a marker for coronary reperfusion and ventricular arrhythmias.

\section{Methods}

One hundred and six patients with reperfusion (WR) and 48 without reperfusion (WtR) who have received thrombolytic therapy in the acute phase of infarction were studied. ECG carried out on admission as well as on day 4 of patient's course were analyzed. $\Delta Q T$ - defined as the difference between maximum and minimum QT interval - was measured by 12-lead ECG.

\section{RESULTS}

The reperfusion group showed significant $\triangle Q T$ reduction - from $89.66 \pm 20,47 \mathrm{~ms}$ down to $70.95 \pm 21.65 \mathrm{~ms}(p<0.001)$. On the other hand, the group without reperfusion showed $\triangle Q T$ significant increase - from $81.27 \pm 20.52 \mathrm{~ms}$ up to $91.85 \pm 24.66 \mathrm{~ms}$ $(p<0.001)$. Logistic regression analysis showed that reduction magnitude beween pre- and post-thrombolysis $\triangle Q T$ was the independent factor to most effectively identify coronary reperfusion (OR 1.045, p<0.0001; Cl 95\%). No significant difference was found in dispersion measures when patients with ventricular arrhythmias were compared with those with no arryhthmias in the course of the first 48 hours.

\section{Conclusion}

The study shows that $\triangle Q T$ is significantly reduced in patients with acute myocardial infarction submitted to successful thrombolysis, and is increased in infarcted patients with closed artery. $\Delta Q T$ reduction between the pre- and post-thrombolysis condition was a predictor for coronary reperfusion of those patients, and did not show correlation to ventricular arrhythmias.

\section{KEY WORDS}

QT dispersion, acute myocardial infarction, coronary reperfusion. 
O conceito de dispersão do intervalo $Q T(\Delta Q T)$ definido como a diferença entre o maior e o menor intervalo QT medidos no ECG de doze derivações foi introduzido por Day e cols. ${ }^{1}$ no início da década de 1990 . Inicialmente foi proposto como um índice de instabilidade elétrica, e representaria a expressão da variação regional fisiológica da recuperação da excitabilidade miocárdica ${ }^{2,3}$.

A partir de então, a análise da dispersão do QT passou a ser aceita com método não-invasivo para detecção da heterogeneidade da repolarização ventricular, sendo um marcador de arritmogênese, especialmente diante de um substrato isquêmico ${ }^{4}$. Além disso, há estudos relacionando-a como índice prognóstico na insuficiência cardíaca e na cardiomiopatia hipertrófica ${ }^{5,6}$.

Há, entretanto, poucos relatos na literatura sobre o efeito da reperfusão precoce na repolarização ventricular de pacientes com infarto agudo do miocárdio (IAM). Estudos em animais indicam que a isquemia regional e a reperfusão alteram a duração do potencial de ação e a velocidade de condução, levando à menor homogeneidade da recuperação ventricular7,8. Alguns estudos demonstraram que a dispersão do QT é maior na fase inicial do infarto agudo do miocárdio, reduzindo com o tempo, nos casos de trombólise com sucesso, podendo permanecer elevada nos pacientes que desenvolveram fibrilação ventricular ${ }^{9-11}$. Com o intuito de estudar 0 efeito da reperfusão precoce da artéria relacionada ao infarto sobre a dispersão do intervalo QT, e seu valor como marcador de reperfusão coronária e de arritmias ventriculares, avaliamos pacientes que receberam terapia trombolítica na fase aguda do infarto.

\section{MÉTodos}

Foram estudados, retrospectivamente, 154 pacientes internados no Instituto do Coração do Hospital das Clínicas da FMUSP com diagnóstico de infarto agudo do miocárdio, submetidos a trombólise durante o período de 1989 a 1992. Os pacientes foram selecionados de um grupo inicial de 220 pacientes consecutivos que haviam recebido trombólise com o ativador tecidual do plasminogênio tecidual (rt-PA) para avaliação da eficácia do agente.

Os critérios para diagnóstico de IAM foram: elevação do segmento ST igual ou maior que 0,2 $\mathrm{mV}$ em pelo menos duas derivações do plano frontal ou igual ou maior que $0,3 \mathrm{mV}$ em duas derivações contíguas do plano horizontal, com ou sem a presença de ondas $Q$ de duração maior que 40 ms, oclusão da artéria coronária relacionada ao infarto (ARI) mantida após infusão intracoronária de 200 mcg de nitroglicerina e níveis de CK-MB duas vezes maior que o normal.

A administração intravenosa do rt-PA foi iniciada na dose de $100 \mathrm{mg}$ dentro das primeiras cinco horas após o início dos sintomas. Após noventa minutos da administração do trombolítico, foram realizadas novas coronariografia e ventriculografia para avaliar o grau de reperfusão da ARI. Em 24 a 48 horas, a coronariografia era repetida para que se avaliasse a patência da ARI.

Os critérios de exclusão desse estudo foram: fibrilação atrial prévia ou aguda (oito pacientes), bloqueios de ramo pelo feixe de His ou distúrbios de condução intraventricular (oito pacientes), traçados de eletrocardiograma (ECG) que não permitiram avaliação satisfatória (22 pacientes), e presença de nova oclusão no cateterismo controle em 48 horas (onze pacientes) ou ausência de perfusão no primeiro cateterismo com patência no segundo exame (dez pacientes).

Os pacientes foram distribuídos conforme critérios angiográficos de reperfusão e de manutenção da patência arterial em dois grupos:

- Com reperfusão (CR): 106 pacientes com reperfusão logo após a trombólise e com manutenção da patência angiográfica da ARI 48 horas após o evento. 0 tempo médio de reperfusão foi de 56,60 $\pm 16,33$ minutos.

- Sem reperfusão (SR): 48 pacientes sem reperfusão após trombólise e sem patência angiográfica da ARI 48 horas após o evento agudo.

Considerou-se critério clínico de reperfusão a presença de pico precoce de CK-MB, ocorrido até 12 horas após o início da infusão do trombolítico.

O diagnóstico de arritmia ventricular baseou-se na presença de fibrilação ventricular (FV) e/ou taquicardia ventricular sustentada (TVS), caracterizada por episódios ectópicos sucessivos com duração superior a trinta segundos, ocorridas nas primeiras 48 horas do infarto.

Os traçados de ECG avaliados foram os realizados na internação do paciente, antes da infusão do trombolítico e no quarto dia de evolução do infarto. Utilizou-se em todos os pacientes o eletrocardiograma computadorizado com conversor analógico-digital do sinal, modelo 4745 Hewlett-Packard, com aquisição simultânea de três derivações. As doze derivações convencionais foram registradas com velocidade de $25 \mathrm{~mm} / \mathrm{s}$ e amplitude de $10 \mathrm{~mm} / \mathrm{Mv}$.

Para melhorar a precisão das medidas, os traçados foram ampliados em duas vezes por uma fotocopiadora, ficando a escala de velocidade equivalente a $50 \mathrm{~mm} / \mathrm{s}$ e amplitude de $20 \mathrm{~mm} / \mathrm{Mv}$. Os traçados foram analisados por observador único sem prévio conhecimento do status da patência da artéria relacionada ao infarto.

A duração do complexo QRS e a dos intervalos RR, QT e JT foram medidas manualmente em cada uma das doze derivações por dois ciclos consecutivos, por intermédio de uma mesa digitalizadora Kurta IS/ONE com resolução de mil pontos por polegada e um programa desenvolvido pela Informática do InCor.

Os intervalos QT foram medidos a partir do início do complexo QRS até o final da onda T. Os intervalos JT, a partir da subtração dos valores do QRS de cada intervalo QT nas doze derivações. Para cada traçado considerou-se o valor máximo médio do intervalo do intervalo QT (QT máximo) e o mínimo médio (QT mínimo). 
O intervalo QT foi corrigido para a freqüência cardíaca segundo a fórmula de Bazett: $Q T C=Q T /$ raiz quadrada de RR. A dispersão do QT $(\Delta Q T)$, a do JT $(\Delta \mathrm{JT})$ e a dispersão do QTc ( $\triangle \mathrm{QTC})$ foram definidas como as diferenças entre os valores máximos e mínimos dos intervalos, expressas em milissegundos (ms).

Avaliação estatística - As variáveis classificatórias foram dispostas em tabelas, contendo freqüências absolutas e relativas nos dois grupos, sendo as proporções comparadas utilizando-se o teste qui-quadrado ou teste de Fisher. As variáveis contínuas como idade, CK-MB, fração de ejeção e duração dos intervalos foram comparadas utilizando-se o teste $t$ de Student (distribuição normal), ou teste nãoparamétrico de Wilcoxon no caso de distribuição anormal.

Os valores de $p<0,05$ foram considerados significantes. Foi utilizado o sistema estatístico SAS.

\section{REsultados}

A análise comparativa das características clínicas entre os grupos CR e SR (tab. 1) mostrou não haver diferença significativa em relação à idade 53,57 + 10,00 e 53,00 $+9,44(p=0,875)$, predomínio do sexo masculino $(p=$ 0,902), localização do infarto agudo do miocárdio, sendo de parede anterior $58,49 \%$ e $45,83 \%$, e inferior $41,41 \%$ e $54,17 \%$, respectivamente $(p=0,144)$, tempo médio entre início da dor e trombólise 4,5 \pm 0,8 horas e 4,8 \pm
$0,9$ horas, respectivamente ( $p=0,124)$ e níveis máximos de CK-MB 116 UI e 93,5 UI ( $p=0,063$ ). Houve uma tendência de maior mortalidade intra-hospitalar no grupo SR, $14,58 \%$ quando comparado ao com reperfusão $4,72 \%(p=0,05)$. Não houve diferença significativa quanto à presença de FV ou TVS na fase aguda, 15,09\% e $16,67 \%$ ( $p=0,803$ ), e de bloqueio atrioventricular total, $5,66 \%$ e $10,42 \%$ ( $p=0,32$ ), nos grupos CR e $\mathrm{SR}$, respectivamente. Não houve diferença significativa entre os grupos CR e SR na distribuição dos fatores de risco para doença arterial coronária.

No grupo CR, o pico precoce de CK-MB esteve presente em $76,42 \%$ dos casos, enquanto nos SR foi encontrado em $27,08 \%$ ( $p<0,001$ ) (fig. 1). A presença de pico precoce apresentou uma especificidade de 72,92\% e sensibilidade de 76,42\% para detectar reperfusão coronária.

Não houve diferença significativa na distribuição das diferentes artérias relacionadas ao infarto entre os dois grupos, como também não houve diferença entre a extensão da doença aterosclerótica e em relação à função ventricular dos pacientes (tab. 1).

Variáveis eletrocardiográficas - Os resultados das medidas do intervalo RR, duração do QRS, dos intervalos QT $T_{\text {máx }}, Q T_{\text {mín }}, Q T c_{\text {máx }}, Q T c_{\text {mín }}, \mathrm{JT}_{\text {máx }}$ e JT $T_{\text {mín }}$ obtidos no grupo com reperfusão não mostraram significativa em relação aos obtidos no grupo sem reperfusão.

\section{Tabela 1 - Distribuição das variáveis angiográficas segundo os grupos com reperfusão e sem reperfusão}

\begin{tabular}{|c|c|c|c|}
\hline & GCR & GSR & \\
\hline Variável & N & N & $\mathbf{P}$ \\
\hline \multicolumn{4}{|l|}{ Artérias relacionadas ao IAM } \\
\hline Coronária direita & $36(33,96 \%)$ & $22(45,83 \%)$ & \multirow{3}{*}{0,336} \\
\hline Circunflexa & $6(5,66 \%)$ & $4(3,77 \%)$ & \\
\hline Ramo interventricular anterior & $62(58,49 \%)$ & $22(45,83 \%)$ & \\
\hline \multicolumn{4}{|l|}{ Comprometimento da doença coronária } \\
\hline Uniarterial & $49(46,22 \%)$ & $22(45,83 \%)$ & \multirow{3}{*}{0,520} \\
\hline Biarterial & $35(33,01 \%)$ & $13(27,08 \%)$ & \\
\hline Triarterial & $22(20,75 \%)$ & $13(27,08 \%)$ & \\
\hline Fração de ejeção do ventrículo esquerdo & $0,63 \pm 0,11$ & $0,61 \pm 0,12$ & 0,492 \\
\hline
\end{tabular}

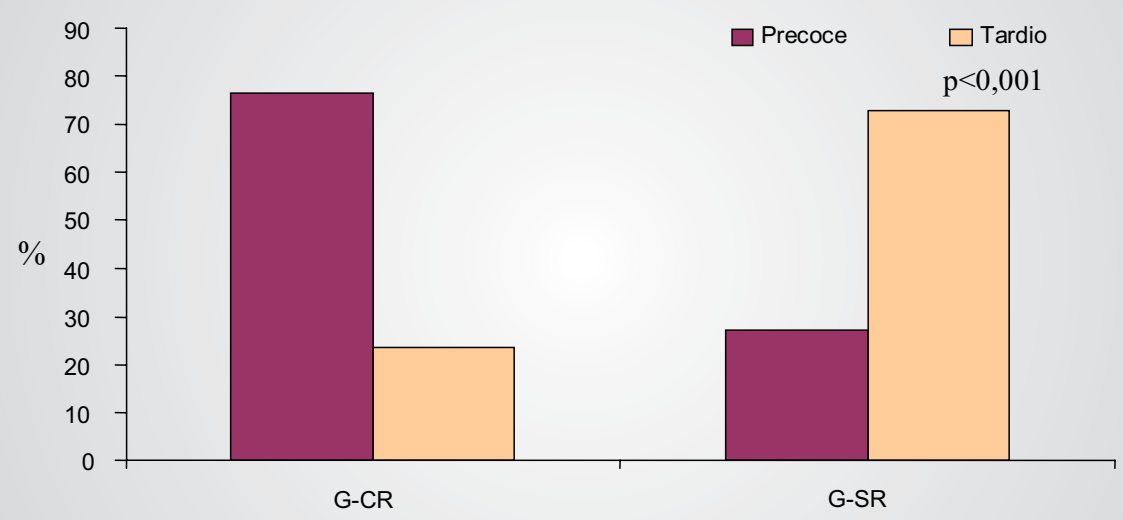

Fig. 1 - Distribuição percentual do pico de CK-MB segundo os grupos com reperfusão e sem reperfusão. 
Duração do QRS, intervalo RR, QT, QTc e JT - Também não se demonstrou diferença significativa entre os resultados das medidas do intervalo $\mathrm{RR}$; da duração do QRS; dos intervalos $Q T_{\text {máx }}, \mathrm{QT}_{\text {mín }}, \mathrm{JT}_{\text {máx }}$ e $\mathrm{JT}_{\text {mín }}$, obtidos no ECG da internação (pré) e no ECG do quarto dia de evolução após a administração do trombolítico (pós) em ambos os grupos. O intervalo $Q T_{\text {máx }}$ se apresentou significativamente maior na situação pré em relação à pós-trombólise, em ambos os grupos ( $p=0,048$ ), e o intervalo $\mathrm{QTC}_{\text {min }}$ se apresentou maior na situação pré em relação a pós-trombólise no grupo sem reperfusão ( $p=$ 0,003) (tab. 2).

Dispersão dos intervalos QT, JT e QTc antes e após a trombólise - Antes da trombólise, os valores dos parâmetros de dispersão dos intervalos QTc ( $\Delta$ QTc) e JT $(\triangle \mathrm{JT})$ não foram diferentes entre o grupo com reperfusão e o sem reperfusão. A variável $\Delta Q T$ se apresentou maior no grupo CR, 89,66 $\pm 20,47$ ms, quando comparada ao grupo SR, $81,27 \pm 20,52 \mathrm{~ms},(p=0,019)$. No quarto dia após trombólise, os valores dos parâmetros de dispersão foram significativamente menores no grupo CR comparado ao grupo SR, respectivamente: $\triangle Q T$ 70,95 $+21,65 \mathrm{~ms}$ e $91,85+24,66 \mathrm{~ms}(\mathrm{p}<0,001), \Delta \mathrm{QTc}$ $84,58 \pm 24,61 \mathrm{~ms}$ e $106,00 \pm 26,48 \mathrm{~ms}(\mathrm{p}<0,001)$; e $\Delta$ JT 77,75 $\pm 24,43 \mathrm{~ms}$ e 92,19 $\pm 24,67 \mathrm{~ms}(\mathrm{p}=$ 0,001) (tab. 3)

\begin{tabular}{|c|c|c|c|c|}
\hline Variável & GCR & GSR & GCR $\times$ GSR & Pré x Pós \\
\hline Ms & Média \pm DP & Média \pm DP & $\mathrm{p}$ & $\mathrm{p}$ \\
\hline \multicolumn{5}{|l|}{$\mathrm{RR}$} \\
\hline Pré & $713,60(197,50-1563,90)$ & $683,30(170,80-1429,60)$ & 0,803 & \multirow{2}{*}{0,465} \\
\hline Pós & $729,70(255,50-1367,70)$ & $736,00(398,9-1642,70)$ & 0,332 & \\
\hline \multicolumn{5}{|l|}{ QRS } \\
\hline Pré & $80,00(60,00110,00)$ & $80(60,00-100,00)$ & 0,683 & \multirow{2}{*}{0,437} \\
\hline Pós & $80,00-(60,00-100,00)$ & $80(60-100)$ & 0,566 & \\
\hline \multicolumn{5}{|l|}{$Q \mathbf{T}_{\text {máx }}$} \\
\hline Pré & $397,9(266,0-620,0)$ & $405,4(292-592)$ & 0,489 & \multirow{2}{*}{0,278} \\
\hline Pós & $396,7(243-538)$ & $398,9(273-642)$ & 0,445 & \\
\hline \multicolumn{5}{|l|}{$Q T_{\text {min }}$} \\
\hline Pré & $315,68 \pm 58,65$ & $326,19 \pm 55,71$ & 0,533 & \multirow{2}{*}{0,078} \\
\hline Pós & $313,27 \pm 53,78$ & $313,92 \pm 59,42$ & 0,533 & \\
\hline \multicolumn{5}{|l|}{ Qtc $_{\text {máx }}$} \\
\hline Pré & $483,5(257,9-820,1)$ & $492,6(358,7-1050,8)$ & 0,360 & \multirow{2}{*}{0,048} \\
\hline Pós & $454,9(275-678,6)$ & $464,7(303,5-627,1)$ & 0,513 & \\
\hline \multicolumn{5}{|l|}{ QTC $_{\text {min }}$} \\
\hline Pré & $379,5(225,1-579,8)$ & $386,4(244,7-931,4)$ & 0,238 & \multirow{2}{*}{$\begin{array}{l}\text { GSR } 0,003 \\
\text { GCR } 0,662\end{array}$} \\
\hline Pós & $370,8(225,0-607,6)$ & $358,7(232,2-501,1)$ & 0,293 & \\
\hline \multicolumn{5}{|l|}{$\mathrm{JT}_{\text {máx }}$} \\
\hline Pré & $317,7(198-560)$ & $334,6(212-512)$ & 0,410 & \multirow{2}{*}{0,305} \\
\hline Pós & $320,9(143-478)$ & $329(183-562)$ & 0,446 & \\
\hline \multicolumn{5}{|l|}{$\mathrm{JT}_{\text {mín }}$} \\
\hline Pré & $238,51 \pm 59,04$ & $248,69 \pm 56,93$ & 0,592 & \multirow{2}{*}{0,084} \\
\hline Pós & $236,66 \pm 53,74$ & $236,21 \pm 60,78$ & 0,592 & \\
\hline
\end{tabular}

. (. - . ) = mediana (mínino-máximo); . \pm . = média \pm desvio padrão; - probabilidade de significância; GCR-grupo com reperfusão; GSR- grupo sem reperfusão.

Tabela 3 - Dispersão do intervalo QT nos grupos pré- e pós-trombólise

$\begin{array}{cccc}\text { Parâmetro }(\mathrm{ms}) & \begin{array}{c}\text { GCR } \\ \text { Média } \pm \text { DP }\end{array} & \begin{array}{c}\text { GSR } \\ \text { Média } \pm \text { DP }\end{array} \\ \text { Pré } & & 81,27 \pm 20,53 & \\ \Delta Q T & 89,66 \pm 20,47 & 99,63 \pm 26,85 & 0,019 \\ \Delta Q T c & 108,18 \pm 28,37 & 81,09 \pm 20,28 & 0,079 \\ \Delta \text { JT } & 83,42 \pm 21,54 & 91,85 \pm 24,66 & 0,528 \\ \text { Pós } & & 106,00 \pm 26,49 & <0,001 \\ \Delta Q T & 70,95 \pm 21,66 & 92,19 \pm 24,67 & 0,001\end{array}$

\pm . = média \pm desvio padrão; GCR- grupo com reperfusão; GSR- grupo sem reperfusão; p- probabilidade de significância; DP- desvio padrão. 


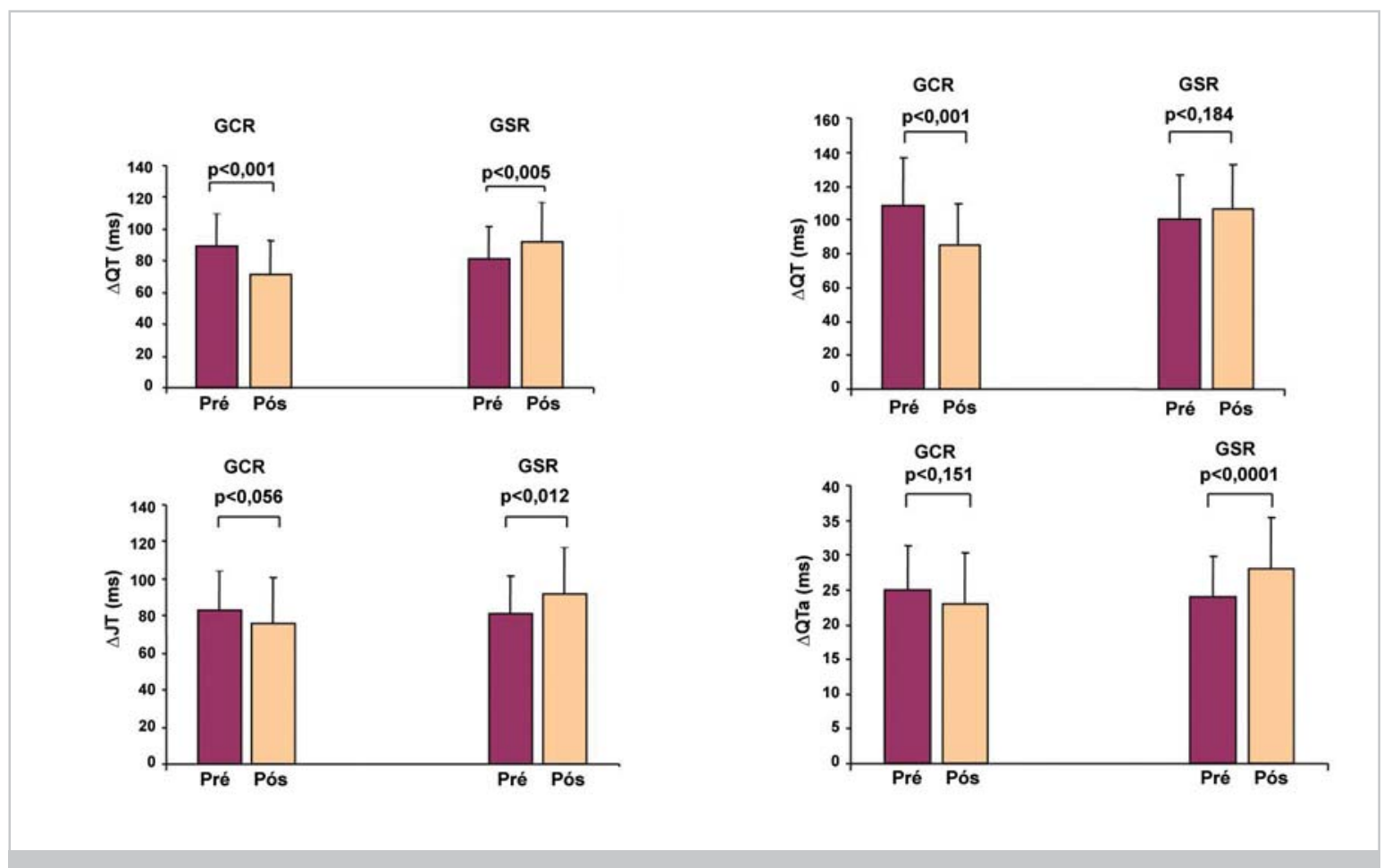

Fig. 2 - Dispersão do intervalo QT, QTc, JT e QTa, nos grupos segundo a condição pré- e pós trombólise.

Na evolução dos pacientes do grupo com reperfusão houve uma redução significativa nas variáveis $\Delta Q T(p<$ $0,001), \Delta Q T c(p<0,001)$ e $\Delta J T(p=0,020)$. Já o grupo sem reperfusão apresentou um aumento significativo nas variáveis $\Delta Q T(p=0,005)$ e $\Delta J T(p=0,022)$ (fig. 2).

Fatores preditivos de reperfusão - Considerando-se as variáveis significativas na análise univariada $\Delta Q T$, $\triangle Q T C$ e $\Delta J T$ pré- e pós-trombólise, pico de CK-MB, classe funcional de Killip $\geq 2$ para reperfusão, ajustouse o modelo de regressão logística com o procedimento de seleção "stepwise". Após o ajuste desse modelo, foi selecionada como fator preditivo de reperfusão a redução da dispersão do intervalo QT obtida após a trombólise, isto é, a diferença entre o QT pré- e o QT pós-trombólise (dif $\Delta Q T$ ) (tab. 4). Assim, quanto maior for a redução desse parâmetro, maior será a probabilidade de reperfusão; ou seja, para cada $1 \mathrm{~ms}$ de diferença encontrada entre o valor de $\Delta$ QT pré- e pós-trombólise, aumenta-se em 1,045 vez a chance de reperfusão (IC 95\%; 1,027-1,064) (fig. 3). A sensibilidade e a especificidade desse modelo são de, respectivamente, $87,74 \%$ e $75 \%$ para uma diminuição de $4 \mathrm{~ms}$, com acurácia de 83,77.

Arritmias ventriculares e variáveis eletrocardiográficas - Não houve diferença significativa das medidas eletrocardiográficas, inclusive as de $\Delta \mathrm{QT}$, quando comparados os pacientes com ou sem arritmias ventriculares nas primeiras 48 horas de evolução do infarto. Embora os grupos não fossem diferentes quanto às características

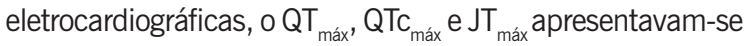
com valores maiores na situação pré-trombólise em relação à pós-trombólise no grupo com arritmia ventricular (tab. 5).

\section{Discussão}

Esse estudo demonstra que na fase aguda do infarto do miocárdio ocorre aumento na dispersão da repolarização ventricular, avaliada pela medida da dispersão dos intervalos QT, QTc e JT. Além disso, a reperfusão e a manutenção da patência da artéria relacionada ao infarto em pacientes submetidos a terapia trombolítica estiveram associadas com menor dispersão dos intervalos QT, QTc e JT, quando comparados aos pacientes sem reperfusão.

Alterações no intervalo QT dependentes do tempo têm sido demonstradas durante a fase aguda do infarto. $O$ aumento da duração e da $\Delta Q T$ ocorre ao longo das primeiras 48 horas do infarto, atingindo valor máximo no terceiro dia ${ }^{12,13}$.

A trombólise e a conseqüente reperfusão também podem afetar a $\Delta Q T$. A reperfusão do miocárdio isquêmico inicialmente aumenta a heterogeneidade da recuperação ventricular induzida pela isquemia, em razão do prolongamento da repolarização ventricular imediatamente após a reperfusão. Porém, posteriormente, os pacientes com melhor grau de reperfusão na artéria relacionada ao infarto evoluem com menor $\Delta Q T^{14}$.

Recentemente, Bonnemeier e cols. ${ }^{15}$ demonstraram que pacientes submetidos a angioplastia por infarto agudo do miocárdico que evoluíam com reperfusão incompleta apresentavam alterações na repolarização ventricular, afetando a dinâmica do QT.

Nossos resultados estão de acordo com os dados encontrados na literatura. Em 1988, Cowan e cols. ${ }^{16}$ descreveram em 42 pacientes pós-infarto do miocárdio 


\begin{tabular}{|cccccc}
\hline \multicolumn{5}{c}{ Tabela 4} & - Valor preditivo da $\Delta \mathbf{Q}$ como parâmetro de reperfusão em análise de regressão logística \\
\hline Variável & Parâmetro estimado & Erro padrão & $\mathbf{P}$ & odds ratio & IC 95\% \\
\hline Intercepto & $-0,5798$ & 0,1990 & - & - & \\
\hline$\Delta Q T$ & 0,0443 & 0,0089 & 0,0001 & 1,045 & $1,027-1,064$ \\
\hline
\end{tabular}

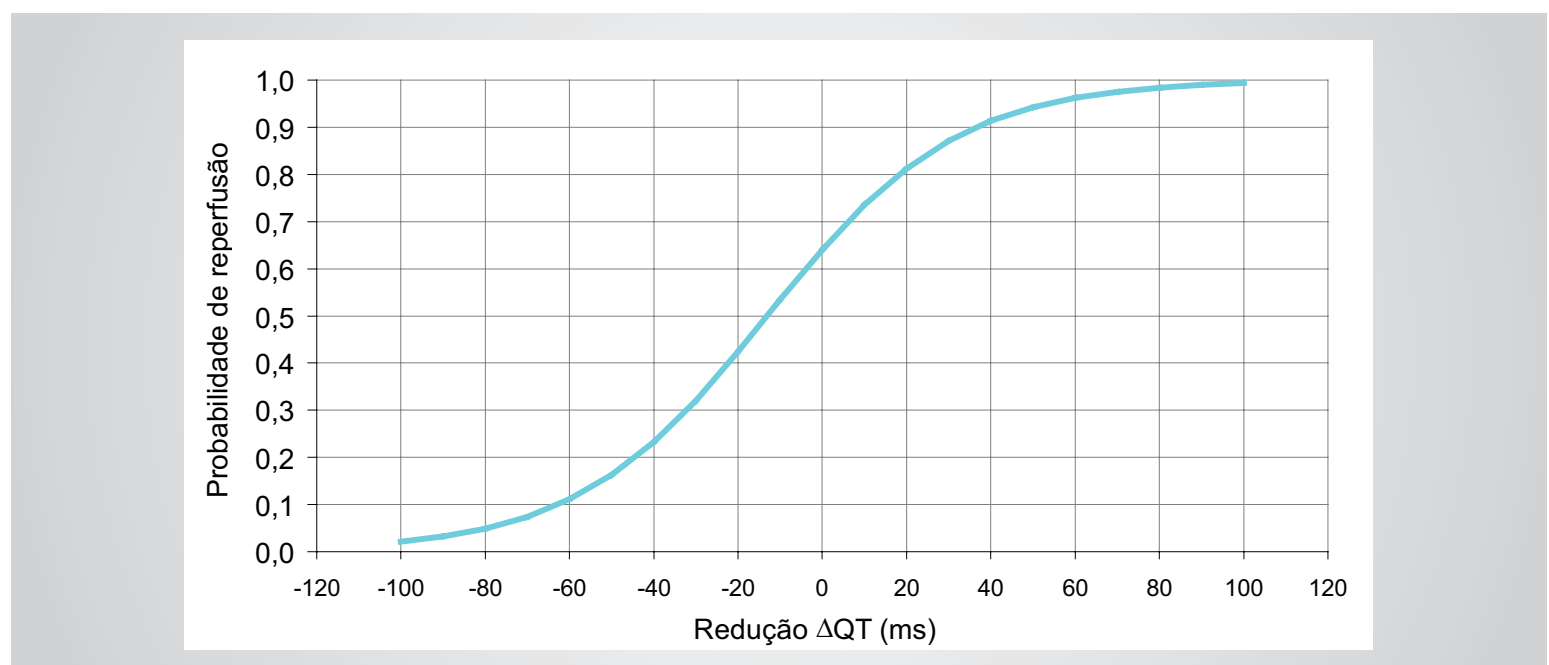

Fig. 3 - Probabilidade de reperfusão coronária através do grau de redução da $\triangle Q T$.

uma maior $\triangle \mathrm{QT}$, quando comparados ao grupo controle. Van de Loo e cols. ${ }^{17}$ demonstraram uma significante redução no $\Delta Q T$ entre a fase aguda e a de convalescença do infarto. Higham e cols. ${ }^{18}$, em 1995 , comparando a $\triangle Q T$ em pacientes com angina e infarto, mostraram que houve redução da $\triangle Q T c$ nesses pacientes na evolução do infarto, sugerindo que isso se devesse à reperfusão obtida pela trombólise com estreptoquinase.

Esses estudos preliminares, entretanto, não correlacionaram essas alterações encontradas na $\Delta Q T$ dos pacientes infartados com o efeito da reperfusão ou não da artéria relacionada ao infarto, como nós demonstramos. Nesse estudo, o comportamento do QT na fase aguda e na convalescença do infarto do miocárdio coincide com a proposição inicial dos estudos clínicos e experimentais, enfatizando que o período após o infarto e o grau de reperfusão da artéria relacionada ao infarto são importantes na determinação da variação da $\Delta Q T$.

Além disso, foi constatado no presente trabalho que os pacientes com infarto agudo apresentam todas as variáveis de dispersão aumentadas e que a recanalização e a patência da artéria relacionada provocaram redução nessas variáveis, quando avaliadas no quarto dia após o evento.

Foi possível demonstrar por meio da análise de regressão logística que, dentre as variáveis relacionadas à reperfusão, a redução da $\Delta Q T$ entre a situação prétrombólise e o quarto dia de evolução foi o parâmetro que melhor identificou a presença de reperfusão coronária.
A patência da artéria relacionada ao infarto se tornou a principal meta na estratégia terapêutica dos pacientes com infarto agudo do miocárdio, pois se sabe que a reperfusão precoce e manutenção da patência arterial são responsáveis pela redução da mortalidade a curto e a longo prazos nos pacientes infartados ${ }^{19,20}$. Portanto, o interesse pela pesquisa de métodos que permitam avaliar de maneira precoce e não-invasiva a reperfusão coronária e a patência arterial é muito importante, por ter implicação prognóstica na evolução do infarto. Esse foi o primeiro estudo cuja metodologia compreendeu a avaliação coronariográfica antes da trombólise, aos noventa minutos e 48 horas após a utilização do rt-PA, o que permitiu correlacionar a variação da $\Delta Q T$ com a reperfusão coronária.

Nesse estudo não se obtive relação entre maior ocorrência de arritmias ventriculares e magnitude da dispersão do QT. Os resultados da literatura são contraditórios, especialmente no que se refere às arritmias da fase aguda. Os dados controversos vêm de estudos com metodologias variadas, como também da dificuldade de análise de um evento causado por múltiplos fatores.

A dispersão do intervalo QT reduz, portanto, significativamente em pacientes com infarto agudo do miocárdio submetidos a trombólise com sucesso, e aumenta significativamente em pacientes infartados que evoluem com artéria fechada. A avaliação da redução da dispersão do QT entre a situação pré- e pós-trombólise é preditora de reperfusão coronária em pacientes infartados submetidos a trombólise. 
Tabela 5 - Comparação das variáveis eletrocardiográficas segundo a ocorrência de arritmias ventriculares

\begin{tabular}{|c|c|c|c|c|}
\hline \multirow[b]{2}{*}{ Ms } & \multicolumn{2}{|c|}{ Arritmias ventriculares } & \multirow{2}{*}{$\begin{array}{c}\mathrm{p} \\
\text { (sim x não) }\end{array}$} & \multirow{2}{*}{$\begin{array}{c}\mathrm{p} \\
\text { (pré x pós) }\end{array}$} \\
\hline & $\operatorname{Sim}(N=23)$ & Não (N = 115) & & \\
\hline \multicolumn{5}{|c|}{ Intervalo RR } \\
\hline Pré- & $805,85(170,75-1091,00)$ & $685,56(296,71-2564,00)$ & 0,315 & \multirow{2}{*}{0,465} \\
\hline Pós- & $753,99(354,62-1210,00)$ & $730,85(255,52-1643,00)$ & 0,846 & \\
\hline \multicolumn{5}{|c|}{ Intervalo QRS } \\
\hline Pré- & $80(60-110)$ & $80(60-110)$ & 0,654 & \multirow{2}{*}{0,437} \\
\hline Pós- & $80(60-100)$ & $80(60-100)$ & 0,565 & \\
\hline \multicolumn{5}{|l|}{$Q T_{\text {min }}$} \\
\hline Pré- & $327,87 \pm 67,63$ & $317,31 \pm 55,91$ & \multirow{2}{*}{0,454} & \multirow{2}{*}{0,198} \\
\hline Pós- & $319,02 \pm 62,71$ & $312,45 \pm 54,15$ & & \\
\hline \multicolumn{5}{|l|}{$\mathbf{Q T}_{\text {máx }}$} \\
\hline Pré- & $416,22(284,00-561,00)$ & $400,20(266,00-620,00)$ & 0,216 & \multirow{2}{*}{0,278} \\
\hline Pós- & $396,67(282,04-498,00)$ & $398,90(243,00-642,00)$ & 0,746 & \\
\hline \multicolumn{5}{|l|}{$Q \mathrm{TC}_{\min }$} \\
\hline Pré- & $392,03(262,58-931,42)$ & $379,69(225,12-579,75)$ & 0,847 & \multirow{2}{*}{0,052} \\
\hline Pós- & $367,59(259,80-607,59)$ & $368,60(225,00-578,33)$ & 0,844 & \\
\hline \multicolumn{5}{|l|}{ QTC $c_{\text {máx }}$} \\
\hline Pré- & $487,89(338,76-1051,00)$ & $484,31(257,88-820,05)$ & 0,648 & \multirow{2}{*}{0,048} \\
\hline Pós- & $466,75(360,28-678,59)$ & $459,09(275,00-673,33)$ & 0,809 & \\
\hline \multicolumn{5}{|l|}{$\mathrm{JT}_{\text {máx }}$} \\
\hline Pré- & $333,61(206,45-501,00)$ & $319,21(198,00-560,00)$ & 0,177 & \multirow{2}{*}{0,305} \\
\hline Pós- & $319,17(182,04-437,00)$ & $321,76(143,00-562,00)$ & 0,629 & \\
\hline \multicolumn{5}{|l|}{$\mathrm{JT}_{\text {min }}$} \\
\hline Pré- & $251,21 \pm 68,26$ & $239,92 \pm 56,51$ & \multirow{3}{*}{0,396} & \multirow{2}{*}{0,246} \\
\hline Pós- & $243,60 \pm 63,90$ & $235,22 \pm 54,39$ & & \\
\hline \multicolumn{4}{|l|}{$\Delta \mathbf{Q T}$} & \\
\hline Pré- & $85,92 \pm 21,99$ & $87,25 \pm 20,64$ & \multirow{2}{*}{0,732} & \multirow{2}{*}{0,019} \\
\hline Pós- & $80,83 \pm 21,06$ & $76,85 \pm 25,17$ & & \\
\hline \multicolumn{5}{|l|}{$\Delta Q \mathrm{TC}$} \\
\hline Pré- & $104,08 \pm 28,69$ & $105,78 \pm 28,10$ & \multirow{3}{*}{0,679} & \multirow{2}{*}{0,003} \\
\hline Pós- & $95,96 \pm 24,33$ & $90,39 \pm 27,50$ & & \\
\hline$\Delta \mathbf{J T}$ & & & & \\
\hline Pré- & $86,62 \pm 20,87$ & $81,97 \pm 21,16$ & 0.768 & 0.409 \\
\hline Pós- & $80,25 \pm 22,12$ & $82,62 \pm 25,94$ & & \\
\hline$\Delta Q \mathrm{QTa}$ & & & & \\
\hline Pré- & $26,65(15,60-37,33)$ & $23,22(11,00-48,47)$ & 0,331 & 0643 \\
\hline Pós- & $24,77(13,96-43,28)$ & $23,35(9,89-45,88)$ & 0,887 & 0,643 \\
\hline
\end{tabular}

\section{Potencial Conflito de Interesses}

Declaro não haver conflitos de interesses pertinentes.

\section{REFERÊNCIAS}

1. Day CP, McComb JM, Campbell RW. QT dispersion: an indication of arrythmia risk in patients with long QT intervals. Br Heart J. 1990; 63: $342-4$

2. Merri M, Benhorin J, Alberti M, Locati E, Moss AJ. Electrocardiographic quantitation of ventricular repolarization. Circulation. 1989; 80:301-8.

3. Butrous GS, Dabbas N, Patel PR, Cochrane T, Camm AJ. Measurement of the QT interval. In: Butrous GS, Swartz PJ. Clinical aspects of ventricular repolarization. London: Farrand Press; 1989: 41-8.

4. Tomassoni G, Pisano E, Gardner kMW, Natale A. QT prolongation and dispersion in myocardial ischemia and infarction. J Electrocardiol. 1998; 30: 187-90.
5. Day CP, McComb JM, Campbell RW. QT dispersion: an indication of arrythmia risk in patients with long QT intervals. Br Heart J. 1990; 63: 342-4.

6. Dristas A, Gilligan D, Nihoyannopoulus P, Oakley CM. Amiodarone reduces $Q T$ dispersion in patients with hypertrophic cardiomyopathy. Intern J Cardiol. 1992; 36: 345-9.

7. Han J, Millet D, Chizzonitti B, Moe GK. Temporal dispersion of recovery of excitability in atrium and ventricle as a function of heart rate. Am Heart J. 1996; 71: 481-7.

8. Kurz RW, Xiao-Lin R, Franz MR. Increased dispersion of ventricular repolarization and ventricular tachyarrhythmias in the globally ischaemic rabbit heart. Eur Heart J. 1993; 14: 1561-71. 
9. Moreno FL, Villanueeva T, Karagounis LA, Anderson JL. Reduction in QT interval dispersion by successful thrombolytic in acute myocardial infarction. Circulation. 1994; 90: 94-100.

10. Sahu P, Lim PO, Rana BS, Struthers AD. QT dispersion in medicine: electrophysiological Holy Grail or fool's gold? QJM 2000; 93 (7): 423-31.

11. Kleber AG, Janse MJ, Van Capelle FJL, Durrer D. Mechanisms and time course of $\mathrm{S}-\mathrm{T}$ and $\mathrm{T}-\mathrm{Q}$ segment changes during acute regional myocardial ischaemia in the pig heart determined by extracellular and intracellular recording. Circ Res. 1978; 42: 603-13.

12. Glancy JM, Garrat CJ, Bono DP. Dynamics of QT dispersion during myocardial infarction and ischemia. Intern J Cardiol. 1996; 57: 55-60.

13. Higham PD, Furniss S, Campbell RWF. QT dispersion and components of the QT interval in ischaemia and infarction. Br Heart J. 1995; 73 : 32-6.

14. Sporton SC, Taggart P, Sutton PM, Walker JM, Hardman. Acute ischaemia: a dynamic influence on QT dispersion. Lancet. 1997; 349: 306-9.

15. Bonnemeier H, Wiegand KH, Bode F, Hartmann F, et al. Impact of infarct-related artery flow on QT dynamicity in patients undergoing direct percutaneous coronary intervention for acute myocardial infarction. Circulation. 2003; 108: 2979-86.

16. Cowan JC, Yusoff KM, Amos PA, Gold AE, Bourke JP, Tansuphaswadikul S, Campbell RWF. Importance of lead selection in QT interval measurement. Am J Cardiol. 1988; 61: 83-7.

17. Van de Loo A, Arendts W, Hohnloser SH. Variability of QT dispersion measurements in the surface electrocardiogram in patients with acute myocardial infarction and in normal subjects. Am J Cardiol. 1994; 74: 1113-8.

18. Higham PD, Campbell RWF. QT dispersion. Br Heart J. 1994; 71 : 508-10.

19. .AIMS Trial Study Group. Long-term effects of intravenous anistreplase in acute myocardial infarction. Final report of the AIMS study. Lancet. 1990; 335: 427-31.

20. Gruppo Italiano per lo Studio della Strepotokinase nell'Infarto Miocardico (GISSI). Long-term effects of intravenous thrombolysis in acute myocardial infarction: final report of the GISSI study. Lancet. 1987; 2: 871-4. 